This is the pre-peer reviewed version of the following article:

Robert Matyáš, Jaromíra Chýlková. Study of TATP: Impact of TATP Sublimation on the Content of Residual Acid in TATP Crystals and on TATP's Properties. Propellants Explosives Pyrotechnics. 04 May 2020.

which has been published in final form at https://doi.org/10.1002/prep.201900375.

This article may be used for non-commercial purposes in accordance with Wiley-VCH Terms and Conditions for Self-Archiving."

This preprint version is available from https://hdl.handle.net/10195/77051 


\title{
Study of TATP: Impact of TATP sublimation on the content of residual acid in TATP crystals and on TATP's properties
}

\author{
Robert Matyášs ${ }^{\star a]}$, Jaromíra Chýlková ${ }^{[b]}$ \\ Dedication ((optional))
}

\begin{abstract}
The impact of four inorganic acids (hydrochloric, sulfuric, nitric and perchloric) and six organic acids (formic, acetic, citric, tartaric, oxalic and ascorbic) on the acid concentration in raw TATP crystals and the behavior of TATP during heating have been studied. The residual acid content in solid TATP depends on the type of acid and on its concentration in the reaction mixture. Increasing the concentration of acid in the reaction mixture leads to a higher acid content in TATP crystals. Sublimation of TATP during storage also has a significant impact on the thermal stability of the residual TATP. Sublimation of TATP gradually increases the content of non-volatile acid in TATP crystals (sulfuric and solid organic acids) while the content of volatile acids (hydrochloric, nitric) does not significantly change during TATP sublimation. The increasing sulfuric acid content in TATP crystals during sublimation decreases its thermal stability and the start of TATP decomposition was observed even at $45^{\circ} \mathrm{C}$. The concentration of relatively volatile hydrochloric and nitric acids does not change significantly in TATP crystals during sublimation and, therefore, the sublimation process does not have an impact on TATP's thermal stability in these cases.
\end{abstract}

\section{Introduction}

Triacetone triperoxide (3,3,6,6,9,9-hexamethyl-1,2,4,5,7,8hexoxonane; TATP) is today one of the most commonly-used improvised explosives for both young chemists and terrorists [13]. It has thus been extensively studied for the past two decades even though, because of its volatility, it has no real potential for industrial use. Most of these studies have focused on an analysis of TATP using a variety of analytical methods, with other research being into its physical, chemical, thermal and explosive properties. In improvised conditions, the explosive is almost exclusively synthesized using Wolfenstein's procedure by the reaction of acetone with hydrogen peroxide in an acidic environment [4]. Residues from the acid used as the catalyst during TATP preparation remain enclosed in TATP crystals even though thorough neutralization and washing of the raw TATP are carried out. Residual acid can be removed only by sublimation or re-

[a] R. Matyáš

Institute of Energetic Materials

University of Pardubice, Faculty of Chemical Technology

Studentska 95, 53210 Pardubice, Czech Republic

*e-mail: robert.matyas@upce.cz

[b] J. Chýlková

Department of Environmental and Chemical Engineering

University of Pardubice, Faculty of Chemical Technology

Studentska 95, 53210 Pardubice, Czech Republic crystallization. However neither sublimation nor re-crystallization is used in improvised conditions and TATP is commonly used in its raw state.

The acid used for TATP preparation has not only an impact on product composition [5-12] or its crystalline phase (polymorphism) $[13,14]$ but also on the intrinsic properties of the TATP. Residual acid in TATP crystals can cause chemical decomposition of TATP causing spontaneous transformation into DADP [15-17]. It has a serious impact on thermal stability of TATP as well. The significant decrease in thermal stability has been observed for TATP prepared using sulfuric and perchloric acids, while hydrochloric and nitric acids do not have an influence on TATP behavior during heating $[18,19]$.

Determining the amount of residual acid in TATP can therefore be important. The isotachophoresis method of determining commonly used inorganic acids has already been published [20]. In this study it was decided to describe the influence of acid concentration in the reaction mixture on the concentration of residual acid in raw TATP crystals. Attention was also focused on organic acids that can be used for TATP preparation.

TATP is a volatile compound and it sublimates at normal temperature [21]. Therefore it can be expected that the concentration of residual acid in TATP crystals can change during such spontaneous sublimation. And the change of acid concentration in TATP crystals can have an impact on TATP's properties. An assessment of this effect is the second aim of this study.

\section{Experimental section}

Caution: TATP is a primary explosive sensitive to all mechanical stimuli and to electric discharge and flame. Therefore synthesis and handling of TATP are dangerous operations that require adhering to safety precaution standards for the handling of primary explosives!!!

\subsection{TATP preparation}

TATP was prepared by the reaction of acetone (p.a., Lach-Ner) with hydrogen peroxide (30\%, p.a., Penta). The preparation procedure was the same as described in reference [18]. The following acids were used as the catalyst: hydrochloric (35\%, p.a., Penta), sulfuric (31\%, p.a., Lach-Ner), nitric (65\%, p.a., Penta), perchloric (70-72\%, p.a., Penta), formic (98\%, pure, Penta), acetic (99\%, p.a., Penta), citric (monohydrate, p.a., Lach-Ner), tartaric (pure, Lach-Ner), oxalic (dihydrate, p.a. Penta) and ascorbic acid (Ph. Eur. 7, Lach-Ner). The amount of acid in the 
reaction mixture is expressed as the molar ratio of acid to acetone $n_{c} / n_{a}$ and this ratio was chosen to be between 2 to 0.01 (depending on the acid's solubility in the reaction mixture). No sample of TATP was re-crystallized after the preparation, just thoroughly washed with distilled water.

\subsection{Liquid chromatography}

An Agilent 1200 series HPLC system was used for analysis of TATP samples. Isocratic separation using a solvent mixture consisting of $60 \%$ acetonitrile and $40 \%$ water with a flow rate of

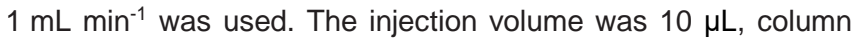
material was BDS Hypersil C-18, particle size $5 \mu \mathrm{m}$, pore size $120 \AA$, column dimensions $250 \mathrm{~mm} \times 4.6 \mathrm{~mm}$, and temperature $30^{\circ} \mathrm{C}$. The chromatograms were evaluated from the absorbance at $210 \mathrm{~nm}$.

\subsection{Analysis of residual acid in TATP crystals}

The acids enclosed in TATP crystals were extracted into water from a TATP toluene solution as described in [20]. The capillary isotachophoresis analytical method was used for determining the type and concentration of anions in the aqueous extracts. The procedure for determining the inorganic acids was the same as described in reference [20]. The instruments and parameters for determining the organic acids were as follows: EA 102 (VillaLabeco, Spišská Nová Ves, Slovakia) was used as isotachophoretic analyzer. This analyzer is equipped with two polytetrafluorethylene capillary columns. Individual zones are detected by a conductivity detector. Analysis was performed in anionic mode for determining formic acid, tartaric acid, oxalic acid and citric acid with a leading electrolyte (LE) containing $10 \mathrm{mmol} / \mathrm{dm}^{3}$ hydrochloric acid, $0.05 \%$ hydroxyethyl cellulose (HEC), with histidine ( $\mathrm{pH} 5.5$ ) and a terminating electrolyte (TE) consisting of $10 \mathrm{mmol} / \mathrm{dm}^{3}$ acetic acid. Separation of acetic acid and ascorbic acid was performed with an electrolyte system consisting of $10 \mathrm{mmol} / \mathrm{dm}^{3}$ hydrochloric acid, $0.05 \%$ hydroxyethyl cellulose (HEC), with histidine $\left(\mathrm{pH} \mathrm{5.92)}\right.$ and $10 \mathrm{mmol} / \mathrm{dm}^{3}$ morpholinoethanesulfonic acid (MES) with tris(hydroxymethyl)aminomethane (TRIS pH 6.54) as terminating electrolyte.

All analyses were performed using a blind scenario for verification of results. Samples of TATP were prepared, and subsequent water extraction carried out, by the synthesizer. The water extracts were then passed to the analyst without revealing any information about synthesis method. In this way the analyst was always ignorant of any expected results. This was the approach used in order to verify the reliability of the method for differentiating the types of acid used for TATP preparation.

\subsection{Differential thermal analysis}

The behavior of TATP during heating was determined using a DTA 550Ex differential thermal analyzer produced by OZM Research. The $30 \mathrm{mg}$ samples were tested in open glass microtest tubes in air. The heating rate was $5^{\circ} \mathrm{C} \mathrm{min}$.

\section{Results and Discussion}

\subsection{Content of residual acid in freshly prepared TATP}

TATP is normally prepared using strong inorganic acids, mostly hydrochloric, sulfuric or nitric acids. This study was enhanced by also using perchloric acid (that was described in the previous study focused on thermal and chemical stability of TATP [18]) and with six organic acids. Each TATP sample was neutralized and thoroughly washed after its preparation to ensure that the TATP sample was not contaminated with residual acid from the reaction mixture.

Based on a previous study, it was expected that the content of residual acid in TATP crystals depended on the acid concentration in the reaction mixture used for preparing the TATP. Therefore TATP was prepared using the above mentioned acids with various concentrations of acid in the reaction mixture. The molar ratio of acid to acetone, $n_{d} / n_{a}$, was used to express the acid concentration in the reaction mixture and isotachophoresis was used as the analytical method for determining the type and content of acid in TATP crystals. Three batches of TATP were prepared using each acid and each molar ratio $n_{c} / n_{a}$, under exactly the same conditions for the purpose of evaluation of the level of batch to batch scatter variation on the results. Results for hydrochloric and sulfuric acids are summarized in Table 1. As can be seen, the scatter in results for one acid and molar ratio is minor in comparison with the impact of the molar ratio $n_{d} / n_{a}$ on the acidity content in TATP crystals for all acids. Similar results were obtained for all other acids.

Table 1. Influence of acidity of reaction mixture (expressed as a molar ratio $n_{d} / n_{a}$ ) on content of residual acid in TATP (in wt. \%) for TATP prepared from hydrochloric and sulfuric acid (three samples were prepared for each acid and molar ratio $n_{d} / n_{a}$ ).

\begin{tabular}{lcccccc}
\hline Molar ratio & \multicolumn{6}{c}{ Concentration of residual acid in TATP (wt. \%) } \\
$n_{d} n_{a}$ & TATP from hydrochloric acid & \multicolumn{2}{c}{ TATP from sulfuric acid } \\
& 1 & 2 & 3 & 1 & 2 & 3 \\
\hline 0.5 & 0.052 & 0.044 & 0.044 & 0.093 & 0.104 & 0.098 \\
0.25 & 0.052 & 0.053 & 0.045 & 0.105 & 0.099 & 0.093 \\
0.10 & 0.018 & 0.024 & 0.016 & 0.051 & 0.051 & 0.059 \\
0.025 & 0.010 & 0.008 & 0.006 & 0.017 & 0.023 & 0.021 \\
0.010 & 0.002 & 0.003 & 0.003 & 0.013 & 0.008 & 0.008 \\
\hline
\end{tabular}


The dependence of the content of residual acid in TATP crystals on molar ratio $n_{c} / n_{a}$ is shown in Figure 1 and Figure 2. The concentration of acid in TATP increases with increasing acidity of the reaction mixture for all acids examined. The specific level of acidity differs and depends on the type of acid.

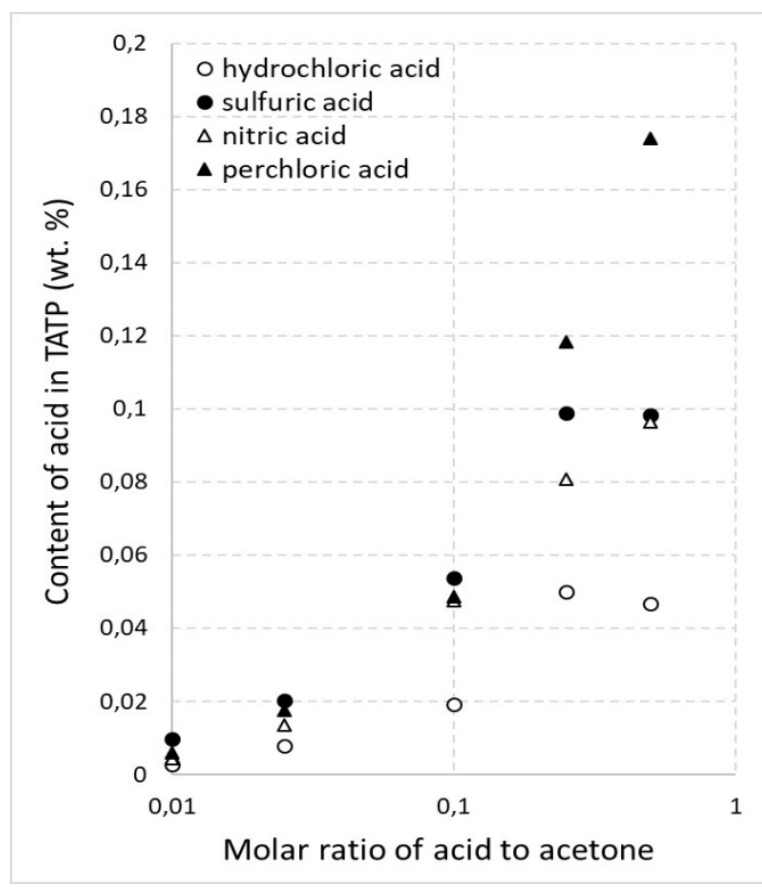

Figure 1. Dependence of residual acid content in TATP crystals on molar ratio of acid to acetone $n_{d} / n_{a}$ for strong inorganic acids (graph presents average value from three experimental values).

The lowest acid content in TATP crystals was observed for TATP prepared using hydrochloric acid while the highest acidity was observed for TATP prepared using perchloric acid. The difference between these two acids is significant, with the acid content for TATP prepared from perchloric acid being 2.2-2.5 times (for $n_{c} / n_{a}$ between 0.01 and 0.25 ) and 3.7 times (for $n_{c} / n_{a}=0.5$ ) higher than that for TATP prepared from hydrochloric acid. The level of acidity for TATP prepared from nitric and sulfuric acids is about the same for each of the molar ratios (except for $n_{d} n_{a}=0.01$ when the acidity content is about 3.5 times higher for TATP from sulfuric acid) and is between the levels for hydrochloric and perchloric acids.

Organic acids are used only rarely for TATP preparation nowadays. The drawback to using such acids is a slower reaction rate leading to formation of large crystals of TATP in the reaction mixture. Moreover, the yield of TATP is significantly lower in comparison with strong inorganic acids. All conditions for organic sample preparation were the same as those in the case of inorganic acids; only the maximum value of ratio $n_{c} / n_{a}$ differs based on the organic acid's solubility in the reaction mixture. The organic acids can be divided into two groups according to residual acidity content in TATP crystals. The highest acid content in TATP crystals was observed for citric and tartaric acids where their content in TATP crystals exceeds even the content of perchloric acid and was 4-11 times higher than the content of hydrochloric acid for the same values of $n_{c} / n_{a}$. The second group consists of formic, acetic, oxalic and ascorbic acids. Their content in TATP crystals is lower than for citric and tartaric acids and on a level with TATP from nitric and hydrochloric acids.

Figure 2. Dependence of residual acid content in TATP crystals on molar ratio

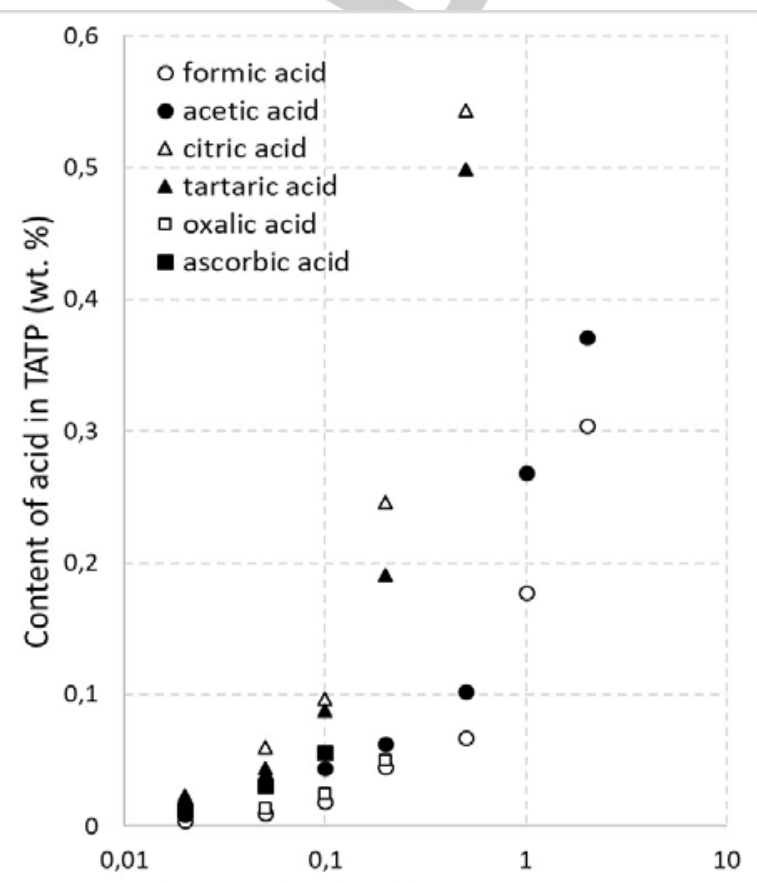

Molar ratio of acid to acetone

of acid to acetone $n_{d} / n_{a}$ for organic acids (graph presents only the average value from three experimental values).

Focus was also on verification of analytical identification of the correct organic acid that was used for TATP preparation. A blind procedure was used for analysis of residual acid in all TATP samples whereby the analyst never new which acid was used for TATP preparation. The type of residual organic acid in TATP was correctly identified in all cases from the position of the ion zones on isotachophoreograms by comparing with the position of the standard zones (examples of isotachophoreograms and more information have been published previously [20]). Therefore identification of the residual acid in TATP crystals can be usable for forensic investigation of TATP to distinguish which acid was used for TATP preparation. The knowledge of the acid used for TATP preparation can be useful for understanding the potential links between samples from crime scenes and the samples related to a specific person.

In the case of TATP samples prepared using citric acid and tartaric acid, focus was on their detection in post-blast residues using isotachophoresis. Traces of these two acids were identified in just a few samples. In addition, a significant concentration of formic acid was detected in all samples. Formic acid, a simple organic molecule, forms in small quantities as a product of detonation of most organic explosives. However, the discovery of residual acid in post-blast residues resulting from TATP has no 
real significance for forensic investigation because small quantities of chlorides, nitrates and sulfates are almost the same in nature as those found in most of the organic acids tested. Thus, it is reasonable to state that their possible presence at an explosion site cannot be considered as conclusive evidence.

3.2 TATP analysis and determination of its behavior during heating for TATP prepared using organic acids

The residual acids in TATP crystals can have a significant impact on TATP's chemical and thermal stability, as has already been published [15-19]. However, these papers only investigated cases with inorganic acids.

Therefore focus was placed on the influence of reaction conditions on the composition of the solid product that forms by reaction of acetone with hydrogen peroxide catalyzed using organic acids. Analysis was carried out using HPLC with UV detection. Two examples of chromatographs are shown in Figure 3. There are two immediate peaks in both chromatograms which represent two conformational isomers of TATP $[22,23]$. TATP forms as a single product for all the TATP samples investigated (all organic acids and all molar ratios $n_{d} / n_{a}$ ).

Unfortunately, organic peroxides do not absorb sufficiently in the UVIVIS spectrum, so it was not possible to detect potential traces of other organic peroxides (especially DADP) that have been described as co-products of TATP. However if they form, they form in very small amounts that cannot be detected using UV/VIS detection.
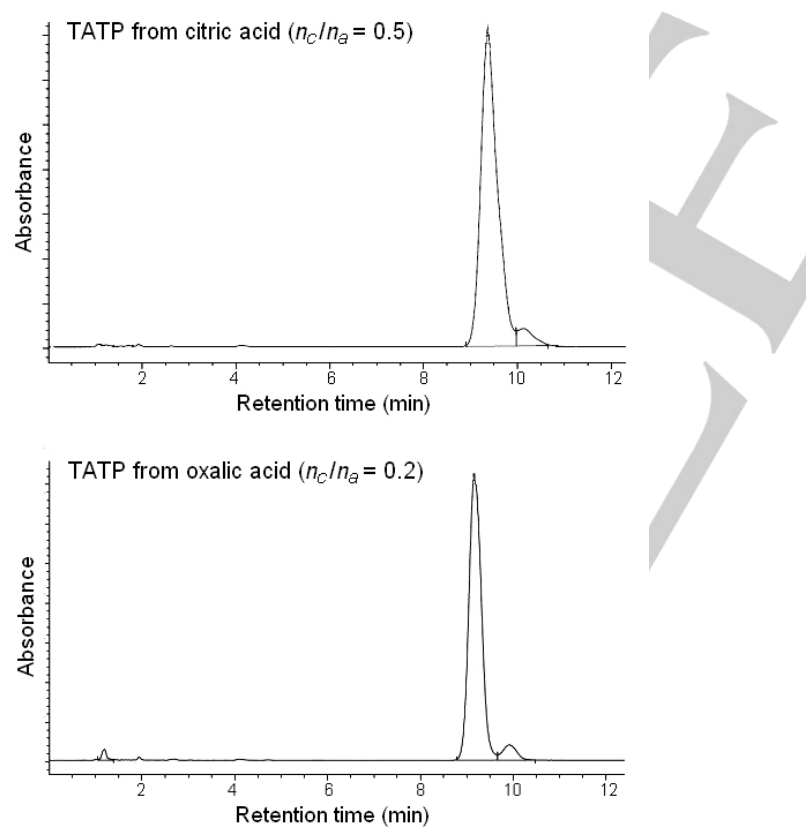

Figure 3. HPLC chromatogram for TATP prepared using citric acid with molar ratio of acid to acetone $n_{c} / n_{a}=0.5$ (upper) and using oxalic acid with molar ratio of acid to acetone $n_{d} / n_{a}=0.2$ (lower).

Behavior of TATP during heating was investigated using DTA. The results of this analysis for TATP prepared using all organic acids for all molar ratios $n_{c} / n_{a}$ are similar to the results for recrystallized TATP. The only difference is in the beginning of the first endothermic peak. The beginning of this endotherm was observed for all freshly prepared samples in the range $76-84^{\circ} \mathrm{C}$. This temperature range is significantly lower than the melting point for pure TATP (which is reported as being between 95$98.8^{\circ} \mathrm{C}$ [24]). In general, the drop in melting point is explained by the presence of impurities. Typically DADP or oligoperoxides are often described as side products of TATP (Sigman et al. [25] reported that the presence of oligoperoxides in TATP lowers the melting point of TATP to $80-91^{\circ} \mathrm{C}$ ). Unfortunately both organic peroxides do not absorb well in UVIVIS so it was not possible to detect their potential trace amounts in TATP. Another explanation of a drop in the melting point is solid state phase transition between TATP polymorphs at $86^{\circ} \mathrm{C}$ suggested by Peterson et al. [26]. Because this study has not focused on this phenomenon, it is not possible to decide which of these two reasons is responsible for the low temperature of the first endotherm for fresh samples of TATP and its moving to higher temperatures during ageing.

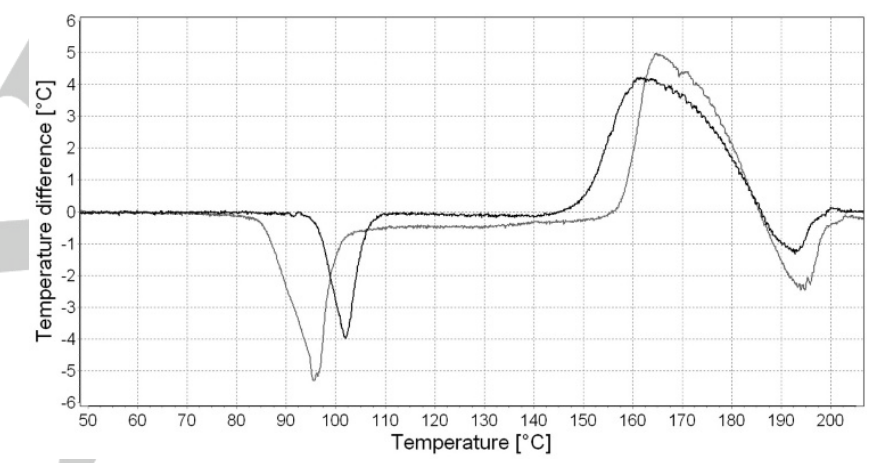

Figure 4. DTA thermogram of TATP prepared using citric acid (molar ratio $n_{d} / n_{a}$ $=0.5$ ). Freshly prepared sample (gray) and the same specimen when $86 \%$ of original weight sublimed off (black).

After melting, TATP is stable in the liquid phase up to a temperature of $144-148^{\circ} \mathrm{C}$ where the start of exothermic decomposition was observed (Figure 4). Generally, TATP prepared using all the organic acids examined shows behavior similar to TATP prepared using hydrochloric or nitric acids.

3.3 Influence of sublimation of TATP on the acid content in TATP crystals

TATP is a volatile compound and sublimates at normal temperature. This causes the concentration of residual acid in TATP crystals to change over time. This phenomenon was investigated for hydrochloric, sulfuric and nitric acids. The molar ratio $n_{d} n_{a}$ for the samples investigated was 0.25 , a commonly used ratio for TATP preparation. TATP samples were placed in a dish at normal temperature and the change of weight of TATP and the concentration of acid in TATP crystals were measured. The results are presented in Figure 5. 
The content of hydrochloric and nitric acid during of TATP sublimation oscillates close to the original value. It means that both TATP and acids sublimate and evaporate eventually. However the concentration of residual sulfuric acid in solid TATP gradually increases as TATP sublimates off. This difference in the behavior during sublimation of TATP is caused by the volatility of the acids. Table 2 contains vapor pressure of the acids mentioned over their aqueous solutions with concentration close to the concentration that was used for TATP preparation.

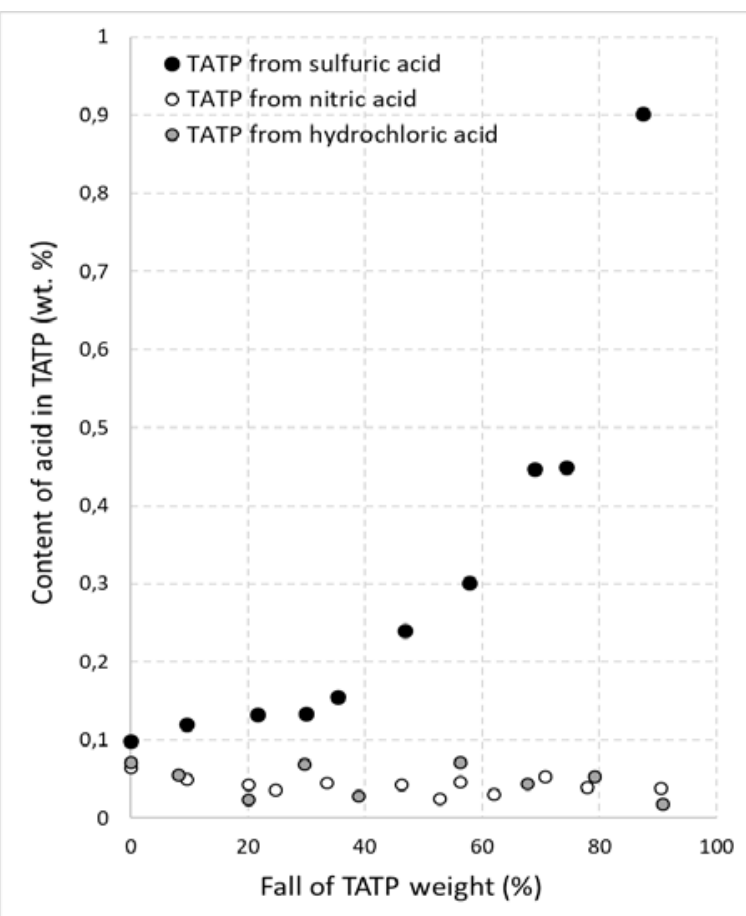

Figure 5. Acid content in TATP crystals following TATP weight loss after sublimation. All TATP samples were prepared using molar ratio $n_{d} / n_{a}=0.25$.

Table 2. Partial pressures of $\mathrm{HCl}, \mathrm{H}_{2} \mathrm{SO}_{4}$ and $\mathrm{HNO}_{3}$ over their aqueous solutions at $20^{\circ} \mathrm{C}$.

\begin{tabular}{lc}
\hline Acid & $\begin{array}{c}\text { Partial pressure over aqueous solution of acid in Pa } \\
\text { (wt. concentration of acid in brackets) }\end{array}$ \\
\hline $\mathrm{HCl}$ & $1.4110^{4}(36 \%)$ \\
$\mathrm{H}_{2} \mathrm{SO}_{4}$ & $1.9310^{-9}(30 \%)$ \\
$\mathrm{HNO}_{3}$ & $2.2410^{2}(65 \%)$ \\
\hline
\end{tabular}

Sulfuric acid is an almost non-volatile substance compared with the other two acids. Therefore it remains in the dish and gradually contaminates the residual TATP crystals during the process of TATP sublimation. The concentration of sulfuric acid can even reach several weight percentage points in solid TATP. The maximum value of sulfuric acid content in solid TATP that was measured was $2 \%$ (sample where $93 \%$ of original TATP weight sublimated off - this value is not presented in the Figure 4). The typical acid burning of paper can be found on the filter paper when
TATP prepared using sulfuric acid (with molar ratio $n_{d} / n_{a}=0.25$ ) is allowed to sublimate on the filter paper (Figure 6).

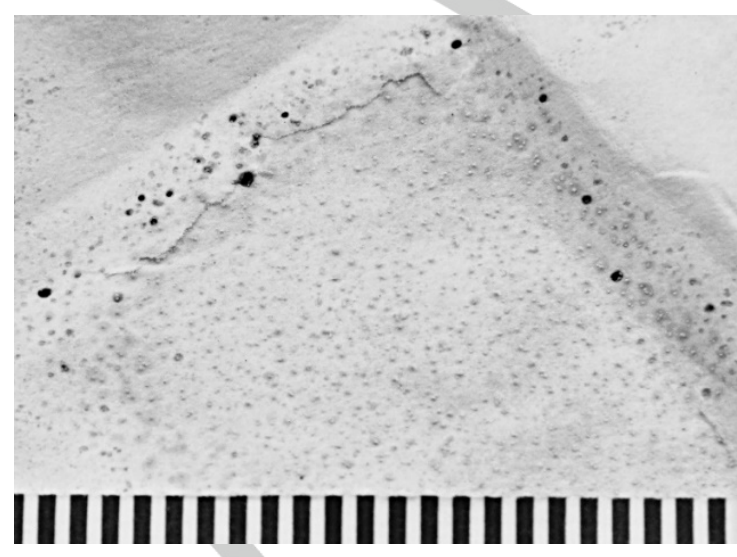

Figure 6. Acid burning on the filter paper. Black and grey spots are places where originally larger TATP crystals were located.

Another parameter that affects the actual concentration of sulfuric acid in the TATP crystals is the original content of the acid in fresh TATP. Therefore three samples of TATP with various molar ratios $n_{d} / n_{a}(0.25,0.1$ and 0.01$)$ were prepared and TATP was freely allowed to sublimate. The dependence of the actual concentration of sulfuric acid in residual TATP crystals during sublimation is demonstrated in Figure 7.

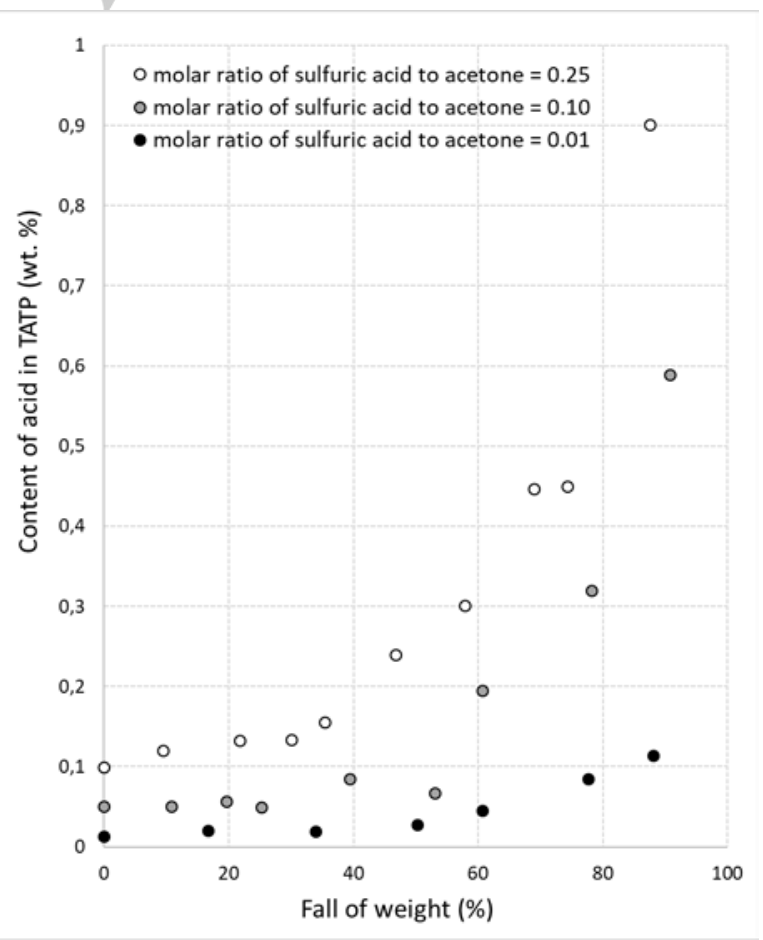

Figure 7. Content of sulfuric acid in TATP crystals depending on the original content and on the drop in weight of TATP following sublimation. 
As can be expected, the concentration of sulfuric acid in solid TATP increases for all of these three samples with just the level of acidity differing based on the original content of acid in the TATP crystals.

3.4 Influence of TATP sublimation on behavior of TATP during heating

The influence of acid enclosed in TATP crystals on the behavior of TATP during heating has already been published $[18,19]$. As has been demonstrated, the acid content can change when TATP spontaneously sublimates depending on the acid concerned. The behavior of TATP during heating (measured by the DTA method) does not change for samples prepared using hydrochloric and nitric acids because the content of these acids in TATP oscillates around the original value during TATP sublimation. However, increasing the content of sulfuric acid in solid TATP during TATP sublimation leads to a decrease in TATP's thermal stability. The negative impact of sulfuric acid on thermal stability of TATP has already been published for freshly prepared samples of TATP [18] but not for aged samples where TATP has partially sublimated. The starting temperature for TATP decomposition generally decreases for solid TATP during the sublimation process. This phenomenon is demonstrated for TATP prepared using sulfuric acid with molar ratio $n_{d} / n_{a}=0.25$ (Figure 8).

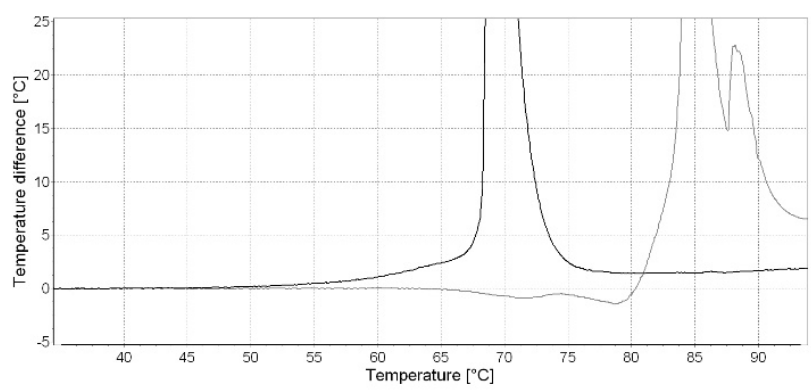

Figure 8. Impact of sublimation of TATP on DTA. TATP was prepared using sulfuric acid with molar ratio $\mathrm{nc} / \mathrm{na}=0.25$. Black curve is for freshly prepared TATP and the grey for aged sample where $93 \%$ of original TATP weight sublimated.

The freshly prepared sample starts to decompose during melting of TATP (overlapping of the second endo peak with the peak of exothermic decomposition, temperature $79^{\circ} \mathrm{C}$ ) while the same, but aged, sample (sample where $93 \%$ of original TATP weight has sublimated) starts to decompose at temperature $45^{\circ} \mathrm{C}$. This temperature was indicated for a $30 \mathrm{mg}$ sample in the heating block of the DTA apparatus. Larger samples of TATP (grams or kilograms) during storage surely start to decompose at lower temperatures as a consequence of the reduction of heat dissipation (heat is generated by the exothermic decomposition of TATP). This behavior could lead to spontaneous explosion of TATP prepared using sulfuric acid during storage in unsuitable conditions at higher temperatures (e.g. in the attic in summer, in a closed car in direct sunlight in summer or during drying near or on a heater).
The organic acids mentioned above are non-volatile solids (except formic and acetic acid). So their concentration in solid TATP increases when TATP sublimates. However the sublimation of TATP does not have a significant impact on the decomposition temperature of TATP for all organic acids examined. The organic acids in this study are weak acids and they do not have a negative impact on thermal behavior of TATP. In all cases, decomposition starts between $144-148^{\circ} \mathrm{C}$.

\section{Conclusions}

TATP is usually prepared by the reaction of acetone with hydrogen peroxide using an acid as catalyst. This acid becomes enclosed in TATP crystals during their formation in the reaction mixture and thus they affect the properties of raw TATP.

The content of these residual acids in TATP crystals highly depends on the acid concerned and on its concentration in the reaction mixture. The higher concentrations of these acids in TATP crystals were found for citric and tartaric acids while the lowest were for hydrochloric, formic and oxalic acids. The differences in acid concentrations in TATP crystals can reach one order of magnitude under the same reaction conditions depending on the acid. Another factor that has an influence on the acid content in TATP crystals is the acid concertation in the reaction mixture, where increasing the concentration of each acid in the reaction mixture leads to an increased concentration of acid in raw TATP.

TATP is a volatile substance that slowly sublimates during storage. This process leads to an increase in acid concentration in TATP crystals for non-volatile acids. While no negative impact of increasing acid concentration in TATP crystals on TATP behavior was observed during heating for weak acids, a significant decrease of thermal stability of TATP crystals prepared using sulfuric acid was detected. The start of TATP decomposition was observed even at $45^{\circ} \mathrm{C}$. Such a low temperature of TATP decomposition can be responsible for unwanted initiation of TATP during storage at slightly higher temperatures - around, say, $50^{\circ} \mathrm{C}$, such as in a car under direct sunlight, in an uninsulated loft in summer, or when drying TATP on or near a radiator.

\section{References}

[1] A. Stambouli, A. El Bouri, T. Bouayoun, M. A. Bellimam, HeadspaceGC/MS detection of TATP traces in post-explosion debris, Forensic Sci. Int. 2004, 146S, S191-S194.

[2] K. Yeager, Trace chemical sensing of explosives, John Wiley \& Sons, New Jersey, 2007.

[3] M. Marshall, J. C. Oxley, Aspects of explosives detection, Elsevier London, 2009.

[4] R. Wolffenstein, Ueber die Einwirkung Wasserstoffsuperoxyd auf Aceton und Mesityloxyd, Ber. Dtsch. Chem. Ges. 1895, 28, 2265-2269.

[5] R. Matyáš, J. Pachman, Study of TATP: Influence of reaction conditions on product composition, Propellants Explos. Pyrotech. 2010, 35, 31-37. 
[6] R. Matyáš, R. Jirásko, A. Lyčka, J. Pachman, Study of TATP: Formation of new chloroderivates of triacetone triperoxide, Propellants Explos. Pyrotech. 2011, 36, 219-224.

[7] M. Fitzgerald, D. Bilusich, Sulfuric, hydrochloric, and nitric acid catalysed triacetone triperoxide (TATP) reaction mixtures: An aging study, J. Forensic Sci. 2011, 56, 1143-1149

[8] M. Fitzgerald, D. Bilusich, The identification of chlorinated acetones in analyses of aged triacetone triperoxide (TATP), J. Forensic Sci. 2012, 57 1299-1302.

[9] J. C. Oxley, J. L. Smith, P. R. Bowden, R. C. Rettinger, Factors influencing triacetone triperoxide (TATP) and diacetone diperoxide (DADP) formation, 40th Annual conference of the north American thermal analysis society, Orlando, USA, 2012, 65-77.

[10] J. C. Oxley, J. L. Smith, P. Bowden, R. Rettinger, Factors influencing triacetone triperoxide (TATP) and diacetone diperoxide (DADP) formation: Part I, Propellants Explos. Pyrotech. 2013, 38, 244-254.

[11] J. C. Oxley, J. L. Smith, L. Steinkamp, G. Zhang, Factors influencing triacetone triperoxide (TATP) and diacetone diperoxide (DADP) formation: Part II, Propellants Explos. Pyrotech. 2013, 38, 841-851.

[12] J. Mbath, D. Knott, S. Steward, D. Cornett, Vapor pressure and sublimation enthalpy of triacetone triperoxide by gas chromatography static headspace approach, Int. J. Energ. Mater. Chem. Propul. 2015, 14 321-329.

[13] O. Reany, M. Kapon, M. Botoshansky, E. Keinan, Rich polymorphism in triacetone-triperoxide, Cryst. Growth Des. 2009, 9, 3661-3670.

[14] G. R. Peterson, W. P. Bassett, B. L. Weeks, L. J. Hope-Weeks, Phase Pure Triacetone Triperoxide: The Influence of Ionic Strength, Oxidant Source, and Acid Catalyst, Cryst. Growth Des. 2013, 13, 2307-2311.

[15] R. Matyáš, J. Pachman, Study of TATP: Spontaneous transformation of TATP to DADP, Propellants Explos. Pyrotech. 2008, 33, 89-91.

[16] R. Matyáš, J. Pachman, Study of TATP: Spontaneous transformation of TATP to DADP, Propellants Explos. Pyrotech. 2009, 34, 484-488.

[17] J. Pachman, R. Matyáš, Study of TATP: Stability of TATP solutions Forensic Sci. Int. 2011, 207, 212-214.

[18] R. Matyáš, J. Pachman, Thermal stability of triacetone triperoxide, Sci. Tech. Energetic Materials 2007, 68, 11-116.

[19] Y.-T. Wu, Y.-S. Wang, J.-J. Horng, C.-M. Chi, Fire and thermal analyses of various fire-extinguishing reagents mixed with triacetone triperoxide (TATP), J. Appl. Fire Sci. 2012, 21, 253-261.

[20] R. Matyáš, J. Chýlková: Study of TATP: Method for determination of residual acids in TATP; Forensic Sci. Int. 2013, 228, 170-173.

[21] H. Ficheroulle, A. Kovache, Contribution à l'étude des explosifs d'amorçage, Meml. Poudres 1949, 31, 7-27.

[22] C. Denekamp, L. Gottlieb, T. Tamiri, A. Tsoglin, R. Shilav, M. Kapon, Two separable conformers of TATP and analogues exist at room temperature, Org. Lett. 2005, 7, 2461-2464.

[23] N. Haroune, A. Crowson, B. Campbell, Characterisation of triacetone triperoxide (TATP) conformers using LC-NMR, Sci. Justice 2011, 51, 50 56.

[24] R. Matyáš, J. Pachman, Primary Explosives, Springer, Heidelberg, 2013.

[25] M. E. Sigman, C. D. Clark, K. Painter, C. Milton, E. Simatos, J. L. Frisch, M. McCormick, J. L. Bitter, Analysis of oligomeric peroxides in synthetic triacetone triperoxide samples by tandem mass spectrometry, Rapid Commun. Mass Spectrom. 2009, 21, 349-356.

[26] P. E. Liley, Physical and chemical data in: Perry's chemical engineers' handbook (Eds.: R. H. Perry, D. W. Green, J. O. Maloney), McGraw-Hill, 1997, pp. 2-14, 2-76, 2-84 